\title{
Feature Extraction and Integration Underlying Perceptual Decision Making during Courtship Behavior
}

\author{
Jan Clemens ${ }^{1,2}$ and Bernhard Ronacher ${ }^{1,2}$ \\ ${ }^{1}$ Behavioral Physiology Group, Department of Biology, Humboldt-Universität zu Berlin and ${ }^{2}$ Bernstein Center for Computational Neuroscience, 10115 \\ Berlin, Germany
}

Traditionally, perceptual decision making is studied in trained animals and carefully controlled tasks. Here, we sought to elucidate the stimulus features and their combination underlying a naturalistic behavior-female decision making during acoustic courtship in grasshoppers. Using behavioral data, we developed a model in which stimulus features were extracted by physiologically plausible models of sensory neurons from the time-varying stimulus. This sensory evidence was integrated over the stimulus duration and combined to predict the behavior. We show that decisions were determined by the interaction of an excitatory and a suppressive stimulus feature. The observed increase of behavioral response with stimulus intensity was the result of an increase of the excitatory feature's gain that was not controlled by an equivalent increase of the suppressive feature. Differences in how these two features were combined could explain interindividual variability. In addition, the mapping between the two stimulus features and different parameters of the song led us to re-evaluate the cues underlying acoustic communication.

Our framework provided a rich and plausible explanation of behavior in terms of two stimulus cues that were extracted by models of sensory neurons and combined through excitatory-inhibitory interactions. We thus were able to link single neuron's feature selectivity and network computations with decision making in a natural task. This data-driven approach has the potential to advance our understanding of decision making in other systems and can inform the search for the neural correlates of behavior.

\section{Introduction}

Decision making in biological systems involves the detection and integration of environmental cues. In neural systems performing a perceptual decision-making task, multiple stimulus cues are extracted by sensory neurons, integrated over some time and combined to inform a motor action (Gold and Shadlen, 2007; Drugowitsch and Pouget, 2012). In this process, two timescales are bridged: first, a short one related to the stimulus features being detected, and second, a longer one corresponding to the integration of evidence derived from multiple feature detectors over the stimulus' time course. This multiscale nature renders the identification of the stimulus cues guiding a decision nontrivial.

On the one hand, the concept of the receptive field developed for sensory neurons has been applied to identify the stimulus features underlying perceptual decisions in artificial tasks (perceptive fields or decision images; Victor, 2005; Neri and Levi, 2006). However, these methods rely on a simple relation between feature and decision. They fail in more naturalistic tasks, e.g.,

\footnotetext{
Received Feb. 17, 2013; revised May 6, 2013; accepted June 18, 2013.

Author contributions: J.C. and B.R. designed research; J.C. performed research; J.C. contributed unpublished reagents/analytic tools; J.C. analyzed data; J.C. and B.R. wrote the paper.

This work was funded by grants from the Federal Ministry of Education and Research, Germany (01GQ0410, 01GQ1001A) and the Deutsche Forschungsgemeinschaft (SFB618, GK1589/1). We thank Olaf Kutzki and Jana Sträter for sharing their behavioral data and Matthias Hennig and Vinzenz Schönfelder for many stimulating discussions. The authors declare no competing financial interests.

Correspondence should be addressed to Jan Clemens, Bernstein Center for Computational Neuroscience, 10115 Berlin, Germany. E-mail: clemensjan@gmail.com.

J. Clemens' present address: Princeton Neuroscience Institute, Princeton University, Princeton, NJ 08544.

DOI:10.1523/JNEUROSCI.0724-13.2013

Copyright $\odot 2013$ the authors $\quad 0270-6474 / 13 / 3312136-10 \$ 15.00 / 0$
}

when the decision is based on integration of evidence over timevarying stimuli.

On the other hand, drift-diffusion and attractor models of decision making well capture the dynamics and inherent tradeoffs of decision making brought about by the long timescales involved in integrating evidence. These models have a thorough grounding in theory and confirmation by experiment (Machens et al., 2005; Uchida et al., 2006; Wang, 2008; Churchland et al., 2011). Yet, they are most often applied in artificial tasks with well controlled, often static and one-dimensional stimuli, where the relevant stimulus cue is known beforehand.

However, decision making in natural tasks relies on stimuli that are dynamic and high-dimensional. Here, we study perceptual decision making during song recognition in grasshoppers as a model of a highly relevant, natural task performed by untrained animals.

During courtship, female grasshoppers of the species Chorthippus biguttulus decide to initiate a courtship ritual based on short temporal features of the male's song (von Helversen and von Helversen, 1997; Ronacher and Stange, 2013). Notably, the female awaits the end of the male's song before signaling its decision. This behavior is thus potentially based on integration of evidence over the duration of the song and the decisive temporal features can occur any time during the song. Due to this integration and due to the time-varying pattern of songs, a successful model needs to appreciate both timescales: the short one associated with the extraction of stimulus features and the long one involved in integrating these features over the stimulus. 
We developed a general and physiologically plausible model of a perceptual decision-making system that we fitted to behavioral data. It combined a feature extraction stage-implemented as a bank of linear-nonlinear models - and an integration stage. This model described the transformation from stimulus to behavioral output very well. In addition, it generalized to a novel stimulus set. Its structure shows what cues guided the female's decision to mate, how these cues were extracted from the song, and how they were combined to yield a perceptual decision.

\section{Materials and Methods}

Structure of the model. We sought a model that could describe the transformation from the raw stimulus to the behavioral response probability with a simple and neurophysiologically plausible structure, which can be fitted to behavioral data and is compatible with theories of optimal decision making.

Many models conceptualize decision making based on sensory stimuli as a two-stage process. First, momentary evidence is accumulated and second, a decision is made based on this evidence, usually once a certain degree of confidence is reached or when the stimulus is over. Since we applied this framework in a natural task without knowing beforehand which stimulus features would be important, we extended this basic structure with a feature detection stage that extracts the evidence from a time-varying stimulus.

Feature detection was implemented by a bank of up to three linear-nonlinear models. The stimulus $s(t)$ was linearly filtered $f_{j}(t)=\int_{-\infty}^{\infty} s(\tau) h_{j}(t-$ $\tau) d \tau$ and subsequently transformed by a sigmoidal nonlinearity $g_{j}(t)=1 /\left(1+\exp \left(-a_{j} f_{j}(t)+b_{j}\right)\right)$. The linear filter constituted the temporal stimulus feature driving decisions. Linear-nonlinear models are physiologically plausible and were successfully used to describe the input-output function of sensory neurons in many sensory systems (Fairhall et al., 2006; Clemens et al., 2012).

The sensory evidence provided by each feature detector was integrated to a feature value. We implemented this step via a perfect integrator $v_{j}=1 / T \int_{0}^{T} g_{j}(t)$. This was proposed by theories of optimal decision making (Beck et al., 2008) and is backed by the finding that neurons in cortex can act as perfect integrators (Churchland et al., 2011).

Finally, the evidence provided by each feature detector and encoded in the feature values was linearly combined to yield a behavioral response value (Gold and Shadlen, 2007; Haefner et al., 2013). In Bayes-optimal decision making these weights are proportional to the reliability of each cue (Drugowitsch and Pouget, 2012). This stage can be implemented in a network with excitatory and inhibitory synapses (Poirazi et al., 2003).

Fitting the model. Often, perceptual decision making is studied in trained tasks where the relevant cues are predetermined by the experimenter. In cases where the stimulus cues are not known beforehand, methods related to the receptive field from neurophysiology have been successfully applied to identify the stimulus features underlying a perceptual decision (Victor, 2005; Neri and Levi, 2006). These methods rely on a tight spatial or temporal correlation between the stimulus feature and the decision. However, the integration step in our model destroys this temporal correlation.

We fitted the model using a genetic algorithm, a class of swarm optimization methods that is inspired by biological evolution (Mitchell, 1998): A population of solutions was randomly initialized. Then, the fitness of each individual solution was evaluated using a goodness-of-fit measure between the predicted and the measured behavioral response. Individual solutions were propagated to the next generation based on their fitness. This way, the best solutions had a higher chance to survive. Variability was introduced to each individual solution through random mutations or recombination of existing solutions. Thereby, good solutions were modified to eventually produce better solutions. This evaluation-selection-mutation cycle was repeated until an optimal solution was found.

This way, the shapes of the filters and the parameters of the nonlinearity were optimized. The weights for combining different feature values were found by linear regression to the behavioral data in the training set. Filters $f_{j}$ were represented as a weighted sum of up to 16 raised-cosine basis functions covering a duration of $64 \mathrm{~ms}$ (Pillow et al., 2008). This sped up training time by reducing stimulus dimensionality and enforced smooth filters. Filters with different durations (see Fig. $1 b$ ) were created by varying the number of basis functions, e.g., the shortest filter $(8 \mathrm{~ms})$ consisted of a single component and was therefore constrained to be unimodal. The longest filter $(64 \mathrm{~ms})$ was built from 16 components. The envelopes of the full ensemble of stimuli in each dataset were normalized to have zero-mean and unit-variance.

Quantification of model performance. We estimated model performance using leave-one-out cross-validation. Generally, cross-validation estimates how well a model predicts novel data not used for fitting (Schönfelder and Wichmann, 2012). This is achieved by dividing the dataset into a training and a test set. In our case the model was trained on 89 of the 90 stimuli. We then calculated the model output for the stimulus left out during training. We repeated this such that each stimulus was used once for testing. Performance was quantified using Pearson's coefficient of correlation $r^{2}$ between the experimental data and the test prediction.

Cross-validation yielded a large set of structurally similar models. To facilitate description of the model structure, we chose to analyze a model trained on the full dataset. This non-cross-validated model exhibited a performance and structure similar to that found with cross-validation: the cross-validated model explained $87 \%$ of the variance in the data, the model trained on the full dataset $91 \%$. This $4 \%$ gain in performance was evenly distributed across all stimuli, leading to a negligible $0.04 \%$ increase per stimulus. Also, the filters of the models fit both ways were highly similar - the median correlation between filters of the full-fits and those of individual cross-validations was 0.98 and 0.87 . Due to this similarity in structure and performance, all the conclusions drawn from the model fitted to the full dataset are valid for the cross-validated models.

Reproducibility of filter shapes across runs. Since the genetic algorithm is a stochastic fitting algorithm, we ensured that the filter shapes were reproducible across different runs. However, this was complicated by two facts: first, the ordering of the filters (filter 1,2, or 3) was arbitrary, and second, the filter shape was only determined up to a global phase shift due to the integration step in the model. We overcame this problem by using filters obtained from fits to the full datasets as templates. For each run, we computed the cross-correlation function (CCF) between each template filter and each of the fitted filters. The peak value of the CCFs indicated the similarity between each filter and each template. We then assigned each filter based on the similarity to the templates. To align the assigned filters, we used the time lag at which the peak correlation occurred. Finally, we calculated the mean aligned filter over all crossvalidation runs and computed the correlation coefficient between this mean and each individual aligned filter. The median correlation was then taken as a measure of filter reproducibility.

Behavioral experiments. A female grasshopper of the species C. biguttulus was placed in a soundproof chamber. Playback of signals and the recording and detection of female response songs was controlled by a computer. All signals in a set were presented 18 times, in randomized order. The probability of the female to respond to the presented stimulus was taken as the behavioral response value for a particular stimulus. This measure was normalized by the response probability to a positive control stimulus interleaved in the normal testing procedure. For further details see Schmidt et al. (2008). Since behavioral data of individual animals were noisy, we used the average responses of 33 or 45 females for each stimulus for fitting. Note that the vast majority of animals conformed to that population average, rendering the model fitted to these averaged data representative of the behavioral preference predominant in natural populations. Some animals deviated systematically from the population average but remained an exception (see Fig. $3 c$ ).

Stimuli. The stimuli on which the model was trained differed in their pattern of amplitude modulations; the carrier spectrum was identical across all datasets and consisted of band-limited white noise with power between 5 and $40 \mathrm{kHz}$ (sampling rate $100 \mathrm{kHz}$ ). The stimulus set consisted of artificial, block-like stimuli constituting a behaviorally very effective abstraction of the natural communication signals of grasshoppers (Fig. 1e).

The song of $C$. biguttulus males consists of a basic subunit: a loud, pulse-like syllable followed by a softer pause. We tested the dependence 

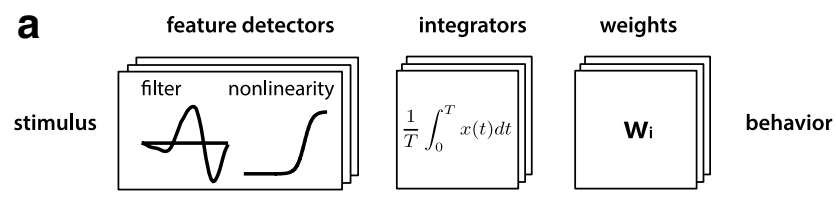

e
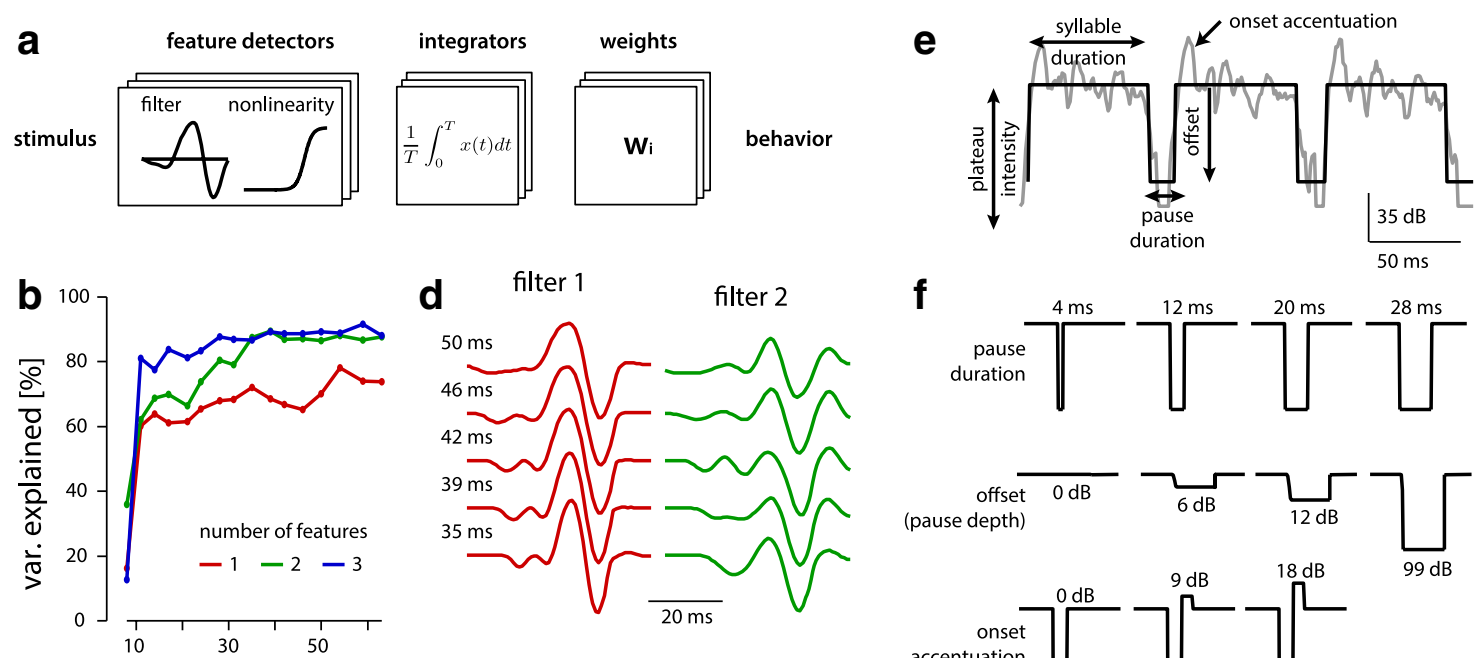

d filter 1

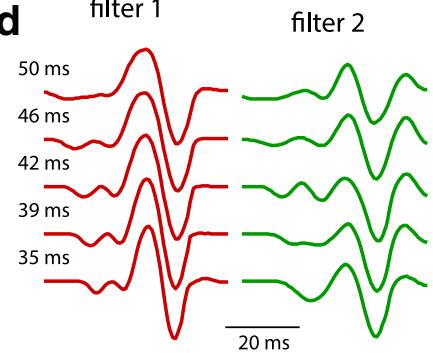

f
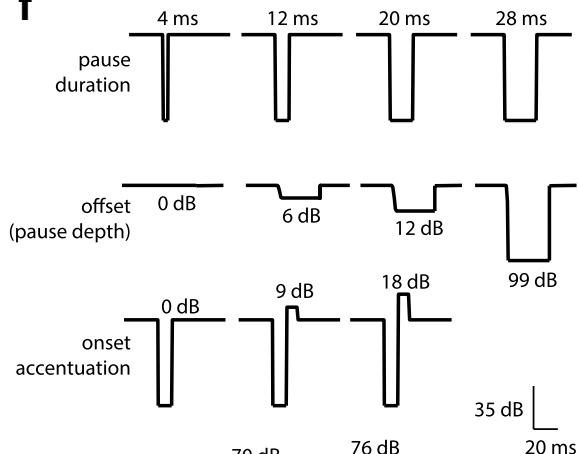

C filter duration $[\mathrm{ms}]$

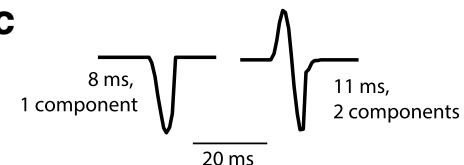

Figure 1. Structure and performance of the model and structure of stimuli. $\boldsymbol{a}$, Layout of our model of a perceptual decision-making system. $\boldsymbol{b}$, Dependence of cross-validation performance on the number of filters and the duration of the associated filters. The filter duration was set by the number of basis components used to represent the filter shapes, i.e., the shortest filter ( $8 \mathrm{~ms})$ consisted of a single basis component, the longest filter ( $64 \mathrm{~ms}$ ) consisted of 16 basis components. $c$, A transition from unimodal (left: single component, $8 \mathrm{~ms}$ ) to bimodal filters (right: two components, 11 $\mathrm{ms}$ ) correlated with a large increase in model performance (performance 0.36 and 0.62 , respectively). This suggests that the detection of transients is fundamental to song recognition in grasshoppers. $\boldsymbol{d}$, Filter shapes were consistent across a wide range of filter durations. Shown are the average filters across cross-validation runs of the two-filter model (first filter: red, left; second filter: green, right) for a range of filter durations. $e$, Envelope of the calling song of a male C. biguttulus (gray) and its reduction to a block stimulus (black). Annotations indicate the signal parameters, which varied in our stimulus set. $f$, A great variety of naturalistic patterns was created by starting from a simple block-like stimulus and changing the pause duration, the depth of the pause, the height of the onset accentuation, and the intensity of the syllable plateau. Onset and offset were defined relative to plateau intensity.

of female responses on four parameters: pause duration, intensity of the syllable plateau, onset accentuation, and offset (Fig. $1 f$ ). Pause duration is given by the gap between adjacent syllables and is a species-specific temporal parameter of male calling songs. Female grasshoppers usually exhibit bandpass tuning for this stimulus feature and prefer the conspecific pause duration (von Helversen and von Helversen, 1997). The other parameters (intensity, onset accentuation, and offset) modulate this tuning for pause. Plateau intensity is defined as the average intensity of the syllable calculated from $10 \mathrm{~ms}$ after the beginning of a syllable to its end. If not stated otherwise, plateau intensity was $70 \mathrm{~dB}$ at the position of the female. Onset accentuations correspond to the difference in amplitude between the loudest point in the first $10 \mathrm{~ms}$ of the syllable and the mean amplitude over the remaining plateau of the syllable. The offset is defined as the drop in amplitude from the end of the syllable to the softest point within the pause. Stimuli were presented in two subsets to two different sets of animals.

In the first subset, the tuning for pause duration at different intensities $(64,70$, and $76 \mathrm{~dB} ; 8$ different pause durations between 4 and $48 \mathrm{~ms})$ and onsets $(0,6$, and $12 \mathrm{~dB} ; 8$ different pause durations between 0 and $48 \mathrm{~ms})$ was tested $(N=34$ animals $)$.

Another subset tested behavioral responses in a full, 3D parameter space: offset $(0,-6,-12$, and $-\infty \mathrm{dB}$ relative to syllable intensity), onset ( 0,9 , and $18 \mathrm{~dB}$ relative to syllable intensity), and pause duration $(4,12,26$, and $38 \mathrm{~ms})$. All possible combinations of parameters yielded 36 different stimuli ( 3 offsets $\cdot 3$ onsets $\cdot 4$ pauses, $N=45$ animals).

Stimuli with gaps were created by using a signal with optimal syllable pause structure ( $80 \mathrm{~ms}$ syllable, $20 \mathrm{~ms}$ pause) and introducing six gaps every $10 \mathrm{~ms}$ starting $10 \mathrm{~ms}$ after the beginning of the syllable and ending $10 \mathrm{~ms}$ before the pause started. Gap duration ranged between 1 and $7 \mathrm{~ms}$. The behavioral data for gap stimuli were taken from Ronacher and Stumpner (1988).

\section{Results}

The model consisted of three stages: a feature extraction stage, an integration stage, and a decision stage (Fig. 1a). The stimulus - in our case the envelope of the song of male grasshoppers-was processed by a bank of up to three feature detectors, which were implemented as linear-nonlinear models. This class of models is used to describe the transformation performed by sensory neurons and constitutes a neurophysiologically plausible implementation of feature detection (Fairhall et al., 2006; Atencio et al., 2008; Geffen et al., 2009; Clemens et al., 2012; Weber et al., 2012). Linear-nonlinear models consist of a linear filter, corresponding to the sensory neuron's preferred temporal feature, and a static nonlinearity, transforming the filtered stimulus to the neural response. The time-varying firing rate of each feature detector is equivalent to the sensory evidence in drift-diffusion type models of decision making. In contrast to standard models of decision making, we derived the structure of each feature detector-the filter and the nonlinearity-and therewith the nature of the sensory evidence directly from behavioral data (see Materials and Methods).

The response of each feature detector was integrated over the full stimulus duration and thereby reduced to a single feature value. Thus, each stimulus was represented by a set of feature values, one for each feature detector. Finally, the feature values from different feature detectors were weighted linearly and summed to predict the response. The model was fitted using a genetic algorithm solely on the basis of the behavioral data without incorporating any prior knowledge as to specific properties of the feature detectors. 


\section{The model successfully reproduced behavior}

We fitted the model to data from the song recognition system of the grasshopper C. biguttulus. The behavioral response values for 90 songs were measured in playback experiments and correspond to the response probability of female grasshoppers-a quantity that is correlated to the attractiveness of a song and to the reproductive success of a male grasshopper (von Helversen and von Helversen, 1997; Klappert and Reinhold, 2003). The calling song of male grasshoppers consists of many repetitions of a basic subunit, a syllable-pause pair. In the experiments, we used artificial, block-like stimuli, which constitute a behaviorally effective abstraction of these natural signals (Fig. 1e,f; von Helversen and von Helversen, 1997). While the duration of the syllable was the same for all stimuli $(80 \mathrm{~ms})$, the duration of the pause ranged from 4 to $48 \mathrm{~ms}$ (Fig. $1 \mathrm{f}$ ). In addition, we also varied the intensity of the syllable plateau and, relative to the plateau intensity, the accentuation at the beginning of the syllable and the offset at the end of the syllable (see Materials and Methods for a definition of these terms).

\section{Two feature detectors maximized model performance}

To determine the number of feature detectors and the duration of the associated filters necessary to explain behavior we assessed the performance of models with up to three feature detectors and filter durations between 6 and $64 \mathrm{~ms}$ using a cross-validation procedure. We varied the filter duration by altering the number of basis elements used for constructing the filters. The shortest filters ( $8 \mathrm{~ms})$ consisted of a single basis element only and were therefore constrained to be unimodal and purely integrating. The next longest filters $(11 \mathrm{~ms})$ were built from two basis elements and had a bimodal, differentiating shape. Interestingly, this coincided with a large (up to 60\%) increase in model performance despite an only modest increase in filter duration (Fig. 1b,c). This suggests that the derivative-like shape of these filters captured an essential aspect of feature extraction-the detection of transients. Further extending the filter duration increased performance for two-filter models by another $30 \%$, but had a weaker impact on one- or three-filter models (19 and $11 \%$ performance increase, respectively). One-filter models of most durations performed worse than two- or three-filter models, while the two-filter models approached the performance of three-filter models $\left(r^{2} \approx\right.$ 0.88 ) for filters longer than $32 \mathrm{~ms}$.

Remarkably, three-filter models exhibited near-optimal performance even for very short filters $\left(r^{2}=0.81\right.$ at a duration of 11 $\mathrm{ms})$. However, the filter shapes were not reproducible across different cross-validation fits (on average 0.36 reproducibility for three filters, each with two components). Two-filter models reached the high performance of three-filter models for filters longer than $32 \mathrm{~ms}$ and exhibited highly reproducible filters across all cross-validation fits (e.g., 0.95 and 0.76 reproducibility for each filter at a duration of $48 \mathrm{~ms}$ ). Also, the general features of both filters' shapes were preserved across a range of durations (35-50 ms; Fig. 1d). This high reproducibility, across individual cross-validation runs and across a wide range of filter durations, indicates that the structure of these models describes relevant aspects of the behavioral preference in grasshoppers. In the following, we therefore described the structure and computational properties of a two-filter model (48 ms duration).

Using a cross-validation scheme we have shown that the model performed well on stimuli that were not used for training. However, these test stimuli contained stimulus features that were in the training set. We next tested whether the fitted model can generalize to predict behavior for a new set of stimuli containing a feature, which was not present in the training data.

One stimulus feature known to be important in guiding song recognition is gap duration (Stumpner and Ronacher, 1994). Natural songs of intact conspecific males exhibit relatively smooth syllable plateaus (Fig. 1e). In contrast, injured males as well as males of other species produce songs with deep and short gaps in the syllable plateau. Songs containing such gaps are rejected by C. biguttulus females (von Helversen, 1972; Kriegbaum, 1989; von Helversen and von Helversen, 1997). We tested the model trained on smooth block-like syllables with stimuli containing gappy syllables and found that it reproduced this rejection of gaps longer than $3 \mathrm{~ms}$ very well (see Fig. $4 a ; r^{2}=0.95$ ). Although gaps are mainly characterized by onsets and offsets, their position within the syllable plateau and their large number renders this feature different from ordinary pauses. The faithful reproduction of the rejection of gaps thus suggests that our model reflected important features of song recognition in grasshoppers.

\section{The model structure revealed the computational principles underlying song recognition in grasshoppers}

Having established that our framework predicted female decisions very well and that it successfully generalized to predict responses to a novel stimulus feature, we next analyzed the model to reveal the song features constituting the cues that guided female decisions and to determine how these cues were combined. To that end, we describe the structure of a two-filter model with $48 \mathrm{~ms}$ long filters and show by which computations it reproduced the behavioral tuning.

\section{The feature detectors were offset detectors}

The filters for the two feature detectors were relatively similar. The first consisted of a positive lobe followed by a negative one (Fig. $2 a$, red). The second feature's filter exhibited an additional positive lobe (Fig. $2 a$, green). Both filters responded best to offsets, that is, a decrease in amplitude (Fig. $2 b$, red and green respectively, downward-pointing arrows). The second filter responded less to the accentuated onset (Fig. 2b, second, downward-pointing arrow) than the first filter. Onsets yielded strongly negative outputs (Fig. $2 b$, upward-pointing arrows). Note that the responses of both filters were shifted in time with respect to each other. This was due to the downstroke in the first filter appearing later than the downstroke in the second filter (Fig. $2 a$, red and green arrows). However, due to the integration step the resulting translation of the output did not affect the feature values. To facilitate comparison of the output of both filters we compensated for this shift in all other figures.

\section{The feature detectors produced binary-like responses}

The output of both filters exhibited a sparse distribution, with most values around zero (Fig. $2 c$, gray shaded area) and a long tail of positive values. This was mainly due to the fact that the filters responded strongly only to transients in the stimulus and most of our stimuli, e.g., those without an onset accentuation, exhibited only few transients. The nonlinearities associated with both filters were very steep, performing a threshold-and-compress operation on the output of each filter, letting pass only the positive tails of the distribution of filter outputs (Fig. 2c). Accordingly, the output of the nonlinearities was almost binary: zero for subthreshold filter values and one for superthreshold filter values (Fig. $2 d$ ).

Integrating the filtered and thresholded stimulus over time yielded feature values (referred to as $f_{1}$ and $f_{2}$ in Fig. 2). Those of the first feature detector correlated strongly with the behavioral 


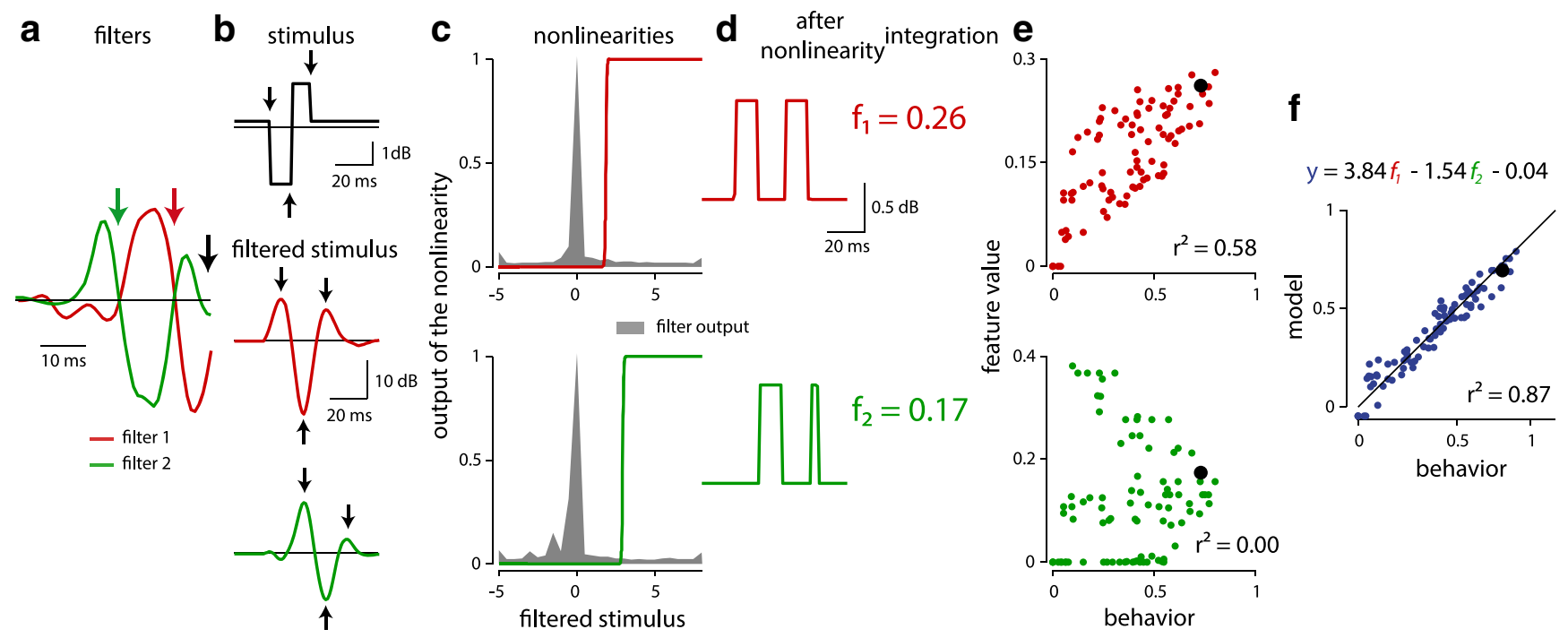

Figure 2. Two-filter model for block-like stimuli. $\boldsymbol{a}$, Filters for both feature detectors (red and green, respectively, duration $48 \mathrm{~ms}$ ). Colored arrows mark the time at which a downstroke in the stimulus is detected, which determines the response phase of the filter. Black arrow indicates the moment of the predicted response relative to the filter. $\boldsymbol{b}$, Example stimulus and response (top, black, pause duration $12 \mathrm{~ms}$, onset $12 \mathrm{~dB}$, offset $18 \mathrm{~dB}$ ). The horizontal black line marks $0 \mathrm{~dB}$. Red and green traces show the stimulus filtered by filter 1 and 2 , respectively. The responses corresponding to onsets and offsets in the stimulus (downward and upward arrows, respectively) are indicated by similar arrows in the filtered stimulus. Note that the responses of the second filter (green) were delayed relative to those of the first filter as the downstrokes (colored arrows in $\boldsymbol{a}$ ) occurred at different phases during each filter. For analysis, the whole stimulus set was normalized to zero mean and unit variance. c, Nonlinearity of the first (top) and second (bottom) feature detector, transforming the output of the filter. Gray areas show distribution of output values of the filters. $\boldsymbol{d}$, The filtered stimulus after applying the nonlinearity. The step-like nonlinearity created pulse-like outputs. Numbers right of each trace indicate the feature values, obtained by averaging the filtered and nonlinearly transformed stimulus over time. $\boldsymbol{e}, \boldsymbol{f}$, Behavioral responses plotted against feature values $(\boldsymbol{e})$ and predicted responses $(\boldsymbol{f})$ obtained by linear combination of both feature values (regression formula shown in $\boldsymbol{f}, f_{1}$, feature value of filter $1 ; f_{2}$, feature value of filter 2 ). Black dots in $\boldsymbol{e}$ and $\boldsymbol{f}$ mark the values for the example stimulus in $\boldsymbol{b}$. $\boldsymbol{r}^{2}$ values show the coefficient of correlation between the behavior and the feature values $(\boldsymbol{e})$ or the predicted response $(\boldsymbol{f})$.

responses $\left(r^{2}=0.58\right.$; Fig. 2e top). Interestingly, the feature values of the second feature did not correlate at all with behavior $\left(r^{2}=\right.$ 0.00 ; Fig. $2 e$, bottom). However, this second feature substantially contributed to model performance, as the linear combination of both filters yielded high correlation values with behavior $\left(r^{2}=\right.$ 0.87; Fig. $2 f)$.

\section{The female decision was the outcome of an excitatory and a suppressive feature}

As a last step in the model, weighting and adding both feature values predicted the behavioral response. The weights determined how each feature influenced behavior. While the first feature had a positive weight of 3.84, the second feature exhibited a negative weight of -1.54 . The bias term was comparatively small $(-0.04)$. As feature values were positive due to the nonlinearity, the first feature encoded stimulus properties increasing responses while the second feature represented stimulus properties decreasing responses. We therefore termed the first feature "excitatory" and the second feature "suppressive." This computation of the decision could be implemented by the interaction of excitatory and inhibitory neurons in a small network.

Thus, behavior was the outcome of combining an excitatory and a suppressive cue. These cues were extracted from the song by offset detectors whose output was thresholded and integrated.

\section{How the model reproduced behavioral preference}

To better understand the role of the excitatory and suppressive cues we next examined how their differential tuning for stimulus parameters reproduced behavior.

\section{The suppressive feature sharpened tuning for pause}

Grasshoppers of the species C. biguttulus exhibit bandpass tuning for pause duration, preferring pause durations between 10 and 20 ms at a syllable duration of $80 \mathrm{~ms}$ (von Helversen and von Helversen, 1997). Very short, as well as very long pauses rarely elicit responses in the majority of animals. As indicated by the high performance of our model (Fig. $1 b$ ), the model very well reproduced this behavior (Figs. $3 b, 4 c, d$ ).

To determine the contribution of the two features in the model to behavioral responses, we quantified their tuning for pause duration (Fig. 3a). Interestingly, the excitatory feature was a high-pass filter for pause duration with a weak bandpass characteristic for pauses longer than $>20 \mathrm{~ms}$, while the suppressive feature was a strict high-pass filter. Thus, suppression was high for long pauses and thereby created the sharp tuning for pause duration with the steep rising and shallower falling flank as seen in the experimental data (Fig. 3a,b).

\section{Differences in feature weights can explain interindividual variability}

Since the model was fitted to the average behavioral data of 33 or 45 animals per stimulus, the results shown so far represent the predominant preference functions observed in natural populations of C. biguttulus. However, few individuals deviated from the population in interesting ways that our model could shed light on. Some animals exhibited only a slowly falling flank for long pauses; others preferred very long pauses (Fig. $3 c$ ). This interindividual variability mapped well to the tuning of the individual features and suggests that different response types might have occurred due to differently weighted features. The behavioral tuning of animals, which exhibited mainly high-pass tuning for pause duration with only a weak bandpass characteristic, was similar to the tuning of the first feature. This suggests little influence (corresponding to a small weight) of the second feature (Fig. $3 a$, green). The tuning of animals preferring long pauses resem- 


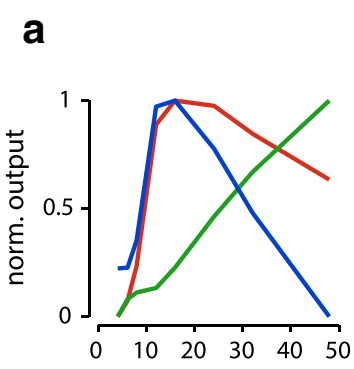

b

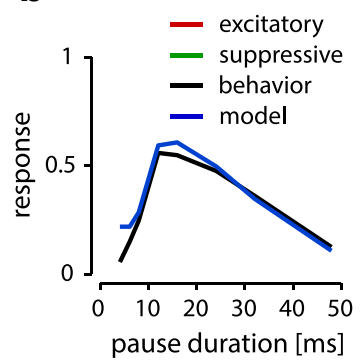

C

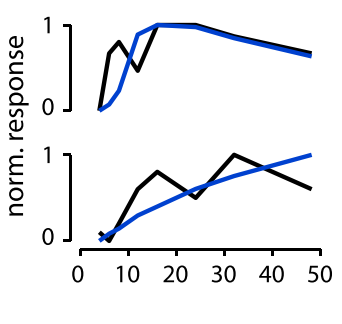

d

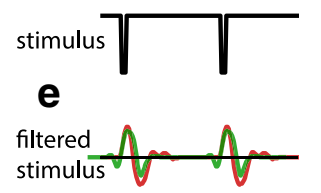

f

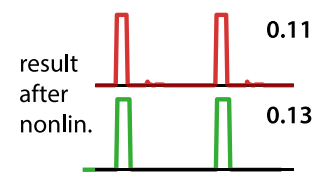

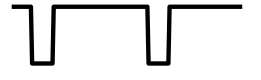
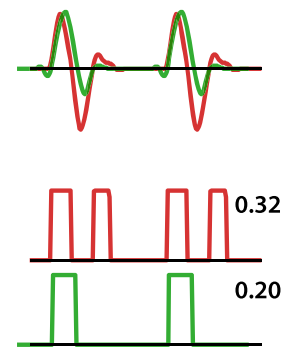

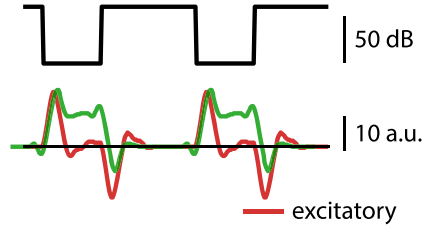

excitatory

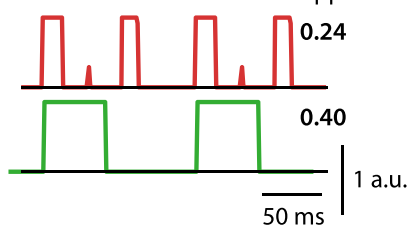

Figure 3. Tuning for pause duration of the feature detectors, the model, and the behavior. $\boldsymbol{a}$, The excitatory feature was a steeply rising, weak bandpass filter for pause duration (red) and the suppressive feature was a high-pass filter with a shallower slope (green). Linear combination of both features yielded a sharp bandpass tuning for pause duration (blue). Tuning curves were normalized to range between 0 and 1 to facilitate comparison. $\boldsymbol{b}$, Pause tuning of the model and behavior (blue and black trace, respectively). c, Pause tuning in individual grasshoppers exhibited weak bandpass tuning resembling that of the first feature (top) or monotonously increasing tuning resembling that of the second feature (bottom). The model could reproduce these interindividual variations by changing the relative weights of both features from [3.84 - 1.54] in the original model to [1 0] and [1 2], respectively (blue traces). d, Block stimuli with 80 ms syllable and 4, 18, or 50 ms pauses. $\boldsymbol{e}$, Stimuli in $d$ filtered with the excitatory and the suppressive filter (red and green, respectively; filters shown in Fig. 2a).f, Output of the excitatory and suppressive feature detector (red and green, respectively) for the stimuli in $\boldsymbol{d}$. Feature values shown to the right of each output trace. represented the rate of pauses and led to the rejection of too short pauses. A suppressive feature encoded the fraction of pauses in the signal and decreased the attractiveness of long pauses.

\section{Rejection of gappy syllables was} established by the suppressive feature Stimuli with gappy syllables were rejected despite having an optimal pause duration (Fig. 4a) and large values of the excitatory feature (Fig. 4b, red). However, since there were so many gaps per syllable, the suppressive feature assumed relatively large values, even though individual gaps elicited only small responses per gap (Fig. $4 b$, green). This was most obvious for gaps longer than $3 \mathrm{~ms}$, since the suppressive features outgrew the excitatory one, which led to a strong decrease in the predicted response. Thus, the suppressive feature conveyed the rejection of stimuli with gaps longer than $3 \mathrm{~ms}$.

\section{The suppressive feature did not act as an intensity gain control}

While the pause duration appears to be an important feature guiding the female decision, the overall intensity of the song can vary considerably depending on the distance between sender and receiver (Clemens et al., 2010). The suppressive feature could act as a simple mechanism for gain control that counteracts any increases of the intensity-related excitatory feature (Carandini and Heeger, 2012).

bled that of the second, suppressive feature. Making the originally suppressive feature excitatory could reproduce this.

\section{Relation between the structure and tuning of the feature detectors}

How did the shape of the filters and the nonlinearities determine the tuning of the feature detectors? Both feature detectors responded only weakly to short pauses (Fig. $3 a$ ). This minimal pause duration was limited by the width of each filter's lobes (compare Figs. $3 d-f$, left, Fig. $2 a$ ).

The differential tuning of both features for long pause durations was due to the different filter shapes and was amplified by the integration process. The excitatory filter responded only transiently to the offset at the end of the pulse (Fig. $3 e$, red). This resulted in a stereotyped response to pause onset and offset for pauses longer than $18 \mathrm{~ms}$ (Fig. $3 f$, red). However, as the number of pause starts and ends per time decreased with increasing pause duration, the feature detector's average activity, its feature value, became smaller for long pauses. This created the weak bandpass tuning for pause duration of the first feature. In contrast, the second filter responded throughout the pause (Fig. $3 e, f$, green). The resulting average feature value was hence proportional to pause duration (Fig. 3a, green).

Thus, while the filter shape governed tuning at short timescales, the integration step was instrumental in shaping the tuning on longer timescales. The bandpass tuning for pause duration was achieved by combining the two features: an excitatory feature
However, although intensity changed the range of preferred pauses only little, louder songs were responded to more strongly in the data as well as in the model (Fig. $4 c, d$ ). The feature values reveal why that was so: while the value of the excitatory feature increased with intensity, the suppressive feature was only weakly affected by intensity (Fig. 4e,f). It could thereby not act as a gain control mechanism by balancing the increase of the excitatory feature with intensity.

\section{Large onset accentuations rendered stimuli without pauses attractive}

Pause duration is considered to be the major stimulus parameter underlying species recognition in the Chorthippus group. The fact that the preference functions of female grasshoppers are tuned to the pause duration found in conspecific songs strengthens this assumption (Fig. 3b; von Helversen and von Helversen, 1994). However, two other parameters of the pause, its depth (offset) and the accentuation at the end of the pause (onset), vary considerably within and across species (Stumpner and von Helversen, 1992; Balakrishnan et al., 2001; von Helversen et al., 2004). We therefore asked how offset and onset shaped the tuning for pause duration.

Interestingly, onset accentuation and offset influenced pause tuning in a complex and sometimes nonlinear manner (Fig. $5 d$ ): the sum of onset and offset needed to exceed $18 \mathrm{~dB}$ to elicit strong responses $>0.5$, suggesting an additive effect of both stimulus parameters (Fig. 5d, diagonal line). Notably, stimuli with no pauses ( $0 \mathrm{~dB}$ offset) were relatively attractive if the onset accen- 


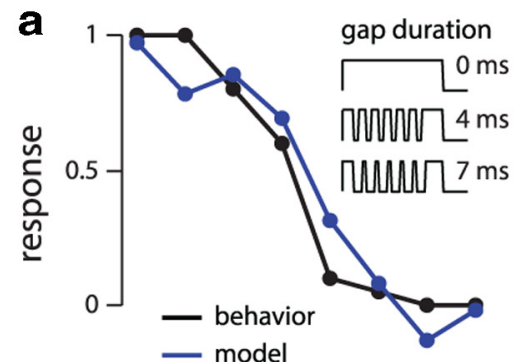

b

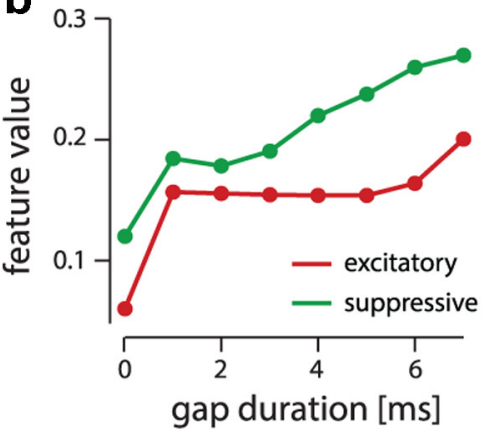

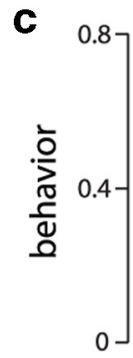

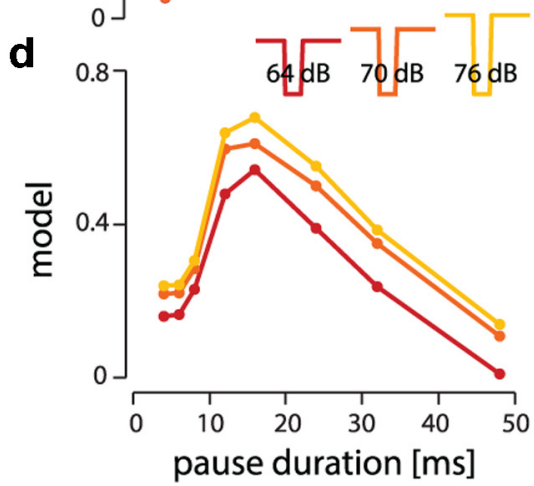

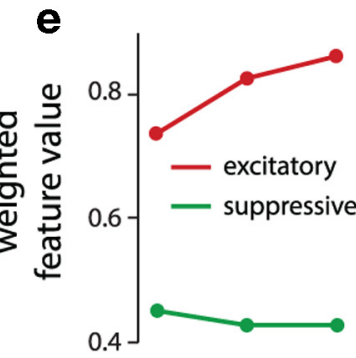

f

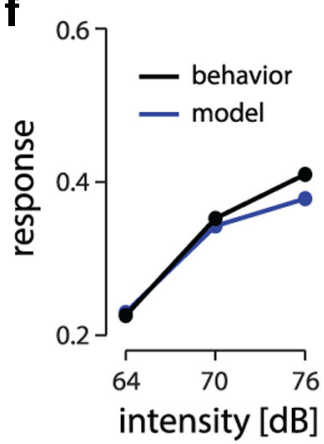

Figure 4. Tuning for gaps and influence of intensity. $\boldsymbol{a}$, Behavioral response (black) and model prediction (blue). The model reproduced behavior very well $\left(r^{2}=0.95\right)$ even though the training set contained no stimuli with gaps in the syllable plateau. $\boldsymbol{b}$, Tuning of both features for gap duration. $\boldsymbol{c}, \boldsymbol{d}$, Pause tuning for block stimuli without accentuated onsets and with plateau intensities of 64,70 , and $76 \mathrm{~dB}$ (see red, orange, and yellow insets for schematic stimuli) in the data (c) and the model (d). $\boldsymbol{e}$, Dependence of feature values (red and green) on plateau intensity for stimuli with a pause duration of $32 \mathrm{~ms}$ and an offset of $99 \mathrm{~dB}$. Shown are the feature's values, scaled by their absolute weight. The excitatory feature (red) increased with intensity, while the suppressive feature (green) was relatively constant. $f$, Predicted and measured response values (blue and black, respectively) for the stimuli used in $\boldsymbol{e}$. Responses increased with intensity.

tuation was strong (Fig. $5 d$, lower right tile, compare Balakrishnan et al., 2001).

Why did onset and offset interact additively for optimal pauses? For optimal pauses $(12 \mathrm{~ms})$, both onset and offset increased the output of both feature detectors (Fig. 5a,b). As both filters responded best to offsets, deeper pauses yielded greater feature values due to the stronger down stroke at the beginning of the pause. Surprisingly, the feature value also increased with onset accentuation. This was counterintuitive as onsets produced negative filter outputs (Fig. $2 b$, upwardpointing arrows). However, onsets also introduced an additional offset at their end (Fig. $2 b$, downward-pointing arrows). Due to the nonlinearity having thresholds greater than zero, only the second, positive effect of the additional offset was reflected in the feature value (compare Fig. $2 d$, second response pulse).

Thus, two properties of the model allowed offsets as well as onsets to increase performance: the invariance of the feature values with respect to when an offset occurred during the syllable and the threshold-like nonlinearity.
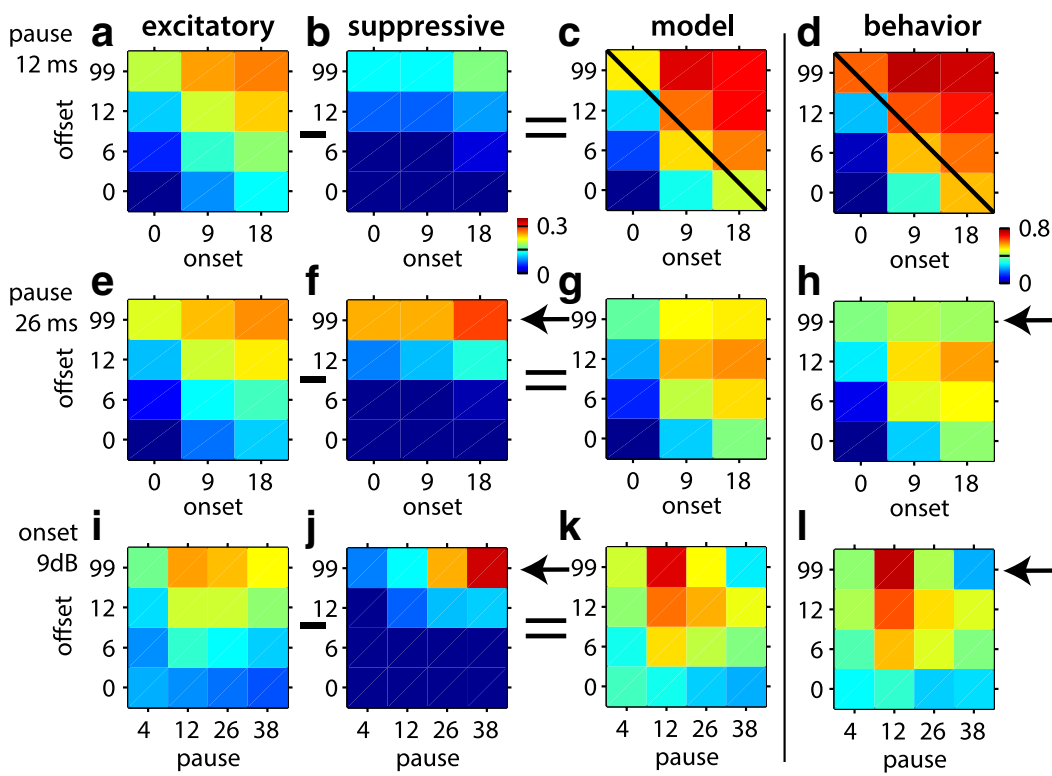

Figure 5. Impact of onset accentuation and offset on pause tuning. Response values are color coded (see color bars). Columns correspond to the tuning of both features, the model, and the experimental data, respectively. Subtracting the suppressive from the excitatory feature yielded the model response. Different rows correspond to different slices of the same, 3D dataset: $\boldsymbol{a}-\boldsymbol{d}$, Dependence of feature values and behavior on onset and offset at an optimal pause of $12 \mathrm{~ms}$. Stimuli for which the sum of onset and offsets exceeds a certain value are responded to strongly $(>0.5$, black line in $\boldsymbol{c}$ and $\boldsymbol{d})$. $\boldsymbol{e}-\boldsymbol{h}, 0$ nset and offset at a long pause of 26 ms. Large values of the suppressive feature (arrow in $\boldsymbol{f}$ ) led to reduced responses for large offsets (arrow in $\boldsymbol{h}$ ). $\boldsymbol{i}-\boldsymbol{l}$, Pause and offset at an onset of $9 \mathrm{~dB}$. At large offsets, tuning of pause duration was narrowest (arrow in $I$ ) due to large values of the suppressive feature (arrow in $j$ ).

\section{Large offsets sharpen tuning for}

\section{pause duration}

Remarkably, the influence of a strong offset ( $99 \mathrm{~dB}$ ) on attractiveness depended on pause duration (Fig. 5, compare $d, h$ ). Offset increased responses for optimal pauses $(12 \mathrm{~ms})$ and reduced responses for long pauses $(26 \mathrm{~ms}$ ) and accentuated onsets (Fig. $5 \mathrm{~h}$, arrow). While this did not alter the preferred pause, it affected the width and height of the tuning curve for pause duration: tuning for pause was widest at $12 \mathrm{~dB}$ offset and strongly accentuated onsets; the tuning curve assumed its greatest peak value and sharpest form at $99 \mathrm{~dB}$ offset for stimuli with onset accentuation 
(Fig. $5 l$, arrow). This increase in sharpness was mainly due to the reduction of attractiveness for long pauses. The model reproduced these interactions well (Fig. $5 g, k$ ).

This sharper tuning for strong offsets was mostly due to the suppressive feature growing faster with offset than the excitatory one, eventually decreasing stimulus attractiveness for large offsets of $99 \mathrm{~dB}$ (Fig. $5 e, j$ and $f, i$, arrows).

\section{Discussion}

We have developed an approach that successfully reproduced female decision making during courtship and generalized to a novel stimulus set (Figs. 1, 4). The model explained behavior as a combination of an excitatory and a suppressive feature. Although these two features were detected by filters that both corresponded most strongly to offsets in the stimulus, they differed in their tuning for individual stimulus parameters (Figs. 2e, 3-5). This differential tuning explained the effect of intensity (Fig. 4) as well as shed light onto the nature of interindividual variability in behavior (Fig. 3c).

\section{Putative neural correlates of the model}

In addition to identifying the features underlying decision our approach also generated a hypothesis of how these features were extracted from the time-varying stimulus. Thus, the linear-nonlinear model can guide the search for neural correlates.

In the grasshopper, third-order auditory neurons have recently been shown to create a specific and diverse representation of grasshopper song (Clemens et al., 2011). Interestingly, these neurons are well described by linear-nonlinear models (Clemens et al., 2012) and some of them encode aspects of the excitatory and of the suppressive feature underlying song recognition.

The excitatory feature was a high-pass filter for pause duration with a slowly decreasing response for longer pauses (Fig. $3 a$, red). The onset-inhibited ascending neurons AN3 and AN4 exhibit similar tuning (Römer and Seikowski, 1985; Krahe et al., 2002). The second, suppressive feature linearly encoded pause duration (Fig. 3a, green). Such tuning is displayed by the ascending neuron AN12 (Creutzig et al., 2009). While AN12's role as an encoder of pause duration was appreciated previously, it was interpreted as an excitatory feature. In contrast, our approach assigned it a suppressive role (compare Creutzig et al., 2010).

The model extracted these features by responses to offsets (Fig. 2). However, electrophysiological evidence suggests that the candidate neurons achieve this tuning in a different way, since they do not respond to offsets but spike phasically or tonically after onsets (Krahe et al., 2002; Creutzig et al., 2009). Further studies are necessary to determine whether the other stimulus parameters in our dataset influence the tuning of these candidate neurons in the same manner as the features in the model.

We emphasize that only the excitatory feature exhibited a considerable correlation with behavior (Fig. 2e). The second feature, although substantially contributing to model performance, did not correlate at all with the full set of behavioral responses. This highlights that a neuron's tuning need not generally correlate with trial-averaged behavior to be behaviorally relevant. Thus, studies seeking neural correlates of behavior are prone to miss important information if they consider each neuron's tuning in isolation. Population-level analyses are thus more suitable to reveal the behavioral impact and importance of a neuron (compare Haefner et al., 2013).

In the model, we have used a perfect integrator. However, a leaky integrator is physiologically more plausible and many models of decision making rely on leaky integration. Given that our stimuli consisted of sequences of identical syllables and that the integration time probably exceeds the duration of an individual syllable (von Helversen, 1972, Ronacher and Krahe, 1998), including leakiness would not affect our results. In the future, signals consisting of mixtures of different syllables could be used to investigate properties of the integrator like integration time and leakiness.

\section{The features underlying species recognition in grasshoppers}

Pause duration is considered the major song feature isolating different members of the Chorthippus species group (Stumpner and von Helversen, 1992; von Helversen and von Helversen, 1994). Curiously, behavioral tuning for pause duration was strongly modified by other stimulus parameters (Fig. 5). Even worse, strongly accentuated onsets rendered songs lacking any pause attractive (Fig. 5d; Balakrishnan et al., 2001). This questions the validity of pause duration as the feature separating grasshopper species.

There are two, not mutually exclusive explanations to resolve this contradiction: either the pause durations of different species are separated well enough to account for the additional variability in the preference functions introduced by offset and onset accentuation found in natural songs (von Helversen and von Helversen, 1994; Hennig et al., 2004; Safi et al., 2006; compare Amézquita et al., 2011) or these additional parameters could be differentially expressed in different species. In the latter scenario, the combinatorial coding of stimulus parameters yields a multidimensional compound feature and may support species separation (compare Wilczynski et al., 1999; Clemens and Hennig, 2013).

\section{The nature of temporal information in communication signals}

In our model, perceptual decisions are based on the integration of sensory evidence provided by short-term feature detectors over the stimulus duration. Thereby, we assume that the exact temporal pattern of the output of feature detectors is not necessary to explain behavior. Such a mode corresponds to "atomized " perception and cannot reproduce Gestalt-like phenomena. This has consequences for the nature of temporal information in grasshopper song as well as in other systems implementing a similar mode of perceptual decision making.

Two competing theories of song recognition have been inspired by the observation that communication signals of many insects exhibit a relatively simple, repetitive structure (von Helversen, 1972; Hoy et al., 1988). However, both theories are not consistent with experimental findings.

The first theory is based on the fact that the rhythmic structure of natural songs yields a parsimonious description of the envelope by a harmonic power spectrum. However, careful experiments testing whether the power spectrum alone was sufficient to explain female selectivity have falsified this hypothesis and demonstrated that the phase spectrum of the song-and hence the song's temporal pattern-does play a decisive role in song evaluation (von Helversen and von Helversen, 1998; Schmidt et al., 2008, compare pitch perception in humans: de Cheveigné, 2005).

The second theory acknowledged that the temporal structure of song was the decisive feature of the song. Exploiting the fact that the song is composed of many repetitions of a simple subunit, it assumed that females employ a Gestalt-like mode of song evaluation, relying on the match of each subunit with an internal, prototype syllable (Hennig et al., 2004). However, a mixture of very different and unattractive subunits can be highly attractive, 
disproving the idea of a static internal template (von Helversen and von Helversen (1998), compare experiments with shuffled song elements in crickets: Pollack and Hoy, 1979; Hennig and Weber, 1997).

These findings suggest that the temporal pattern of the stimulus does play a role, but that the precise sequence of that pattern on a global scale is not important. Our model reconciles these facts by defining temporal features on a short timescale by the shape of the linear filters while discarding the timing of those features within a signal through the averaging operation (Fig. 2d). In the model as in behavior the local phase spectrum - the filter's shape-strongly affects signal attractiveness while the global phase of a feature-its timing within the song — has no impact on behavioral responses.

This mode of perceptual decision making has consequences for how a neuronal representation of behaviorally relevant features should be organized. In many systems, the temporal fidelity of the neuronal responses decreases as one ascends a neuronal pathway while the specificity of responses increases (Joris et al., 2004). This is also the case in grasshoppers, where the representation is very precise and generic in the periphery and becomes less precise and more specific at the level of higher order neurons (Machens et al., 2001; Vogel et al., 2005; Clemens et al., 2011). The system seems to trade information of the "when" of temporal features - their timing - for the "what" of temporal featurestheir specific shape. Since timing information is discarded in the averaging step of our model (Fig. 2), there is no need to sustain a precise timing of spikes in higher order neurons. Temporal precision being energetically costly (Niven et al., 2007), this is an efficient implementation of a perceptual decision-making system.

\section{Conclusion and extensions}

We used the simple and innate acoustically driven behavior of grasshoppers to demonstrate the power of a model for decision making. We explained behavior not in terms of abstract stimulus parameters but based on a plausible mode of feature extraction. This enabled a simple, parsimonious description of behavior and yielded a "natural" stimulus space, which is likely to better match the feature space in which song production and recognition might have evolved (Ryan and Getz, 2000; Geisler and Diehl, 2003; Ryan and Rand, 2003; Akre et al., 2011).

Since our approach is general, it can provide a toolbox to compare feature detection and combination across different species, different tasks or training paradigms, or to develop normative theories of perceptual decision making (Geisler and Diehl, 2003; Beck et al., 2008; Geisler et al., 2009).

Recently, we have applied this framework to behavioral data from two species of crickets seeking to elucidate general principles of song recognition (Clemens and Hennig, 2013). This yielded filters similar to Gabor filters, which are found in early acoustic and visual areas. These Gabor filters allowed us to reproduce a large variety of known preference functions found in insects and anurans. In the present paper, we used the framework to reproduce and describe the behavioral preference for a single species in more detail. The filters found in this paper also resembled Gabor filters. Interestingly, this class of filters has been implied in redundancy reduction and efficient coding in the visual and auditory system (Smith and Lewicki, 2006). The fact that behavioral filters in grasshoppers fall into the same class suggests that grasshoppers implement an efficient code for features of song (Clemens et al., 2011).

\section{References}

Akre KL, Farris HE, Lea AM, Page RA, Ryan MJ (2011) Signal perception in frogs and bats and the evolution of mating signals. Science 333:751-752. CrossRef Medline

Amézquita A, Flechas SV, Lima AP, Gasser H, Hödl W (2011) Acoustic interference and recognition space within a complex assemblage of dendrobatid frogs. Proc Natl Acad Sci U S A 108:17058-17063. CrossRef Medline

Atencio CA, Sharpee TO, Schreiner CE (2008) Cooperative nonlinearities in auditory cortical neurons. Neuron 58:956-966. CrossRef Medline

Balakrishnan R, von Helversen D, von Helversen O (2001) Song pattern recognition in the grasshopper Chorthippus biguttulus: the mechanism of syllable onset and offset detection. J Comp Physiol A 187:255-264. CrossRef Medline

Beck JM, Ma, WJ, Kiani R, Hanks T, Churchland AK, Roitman J, Shadlen MN, Latham PE, Pouget A (2008) Probabilistic population codes for Bayesian decision making. Neuron 60:1142-1152. CrossRef Medline

Carandini M, Heeger DJ (2012) Normalization as a canonical neural computation. Nat Rev Neurosci 13:51-62. CrossRef Medline

Churchland AK, Kiani R, Chaudhuri R, Wang XJ, Pouget A, Shadlen MN (2011) Variance as a signature of neural computations during decision making. Neuron 69:818-831. CrossRef Medline

Clemens J, Hennig MR (2013) Computational principles underlying song recognition in insects. J Comput Neurosci. Advanced online publication. Retrieved Feb. 2013. doi:10.1007/s10827-013-0441-0. CrossRef

Clemens J, Weschke G, Vogel A, Ronacher B (2010) Intensity invariance properties of auditory neurons compared to the statistics of relevant natural signals in grasshoppers. J Comp Physiol A Neuroethol Sens Neural Behav Physiol 196:285-297. CrossRef Medline

Clemens J, Kutzki O, Ronacher B, Schreiber S, Wohlgemuth S (2011) Efficient transformation of an auditory population code in a small sensory system. Proc Natl Acad Sci U S A 108:13812-13817. CrossRef Medline

Clemens J, Wohlgemuth S, Ronacher B (2012) Nonlinear computations underlying temporal and population sparseness in the auditory system of the grasshopper. J Neurosci 32:10053-10062. CrossRef Medline

Creutzig F, Wohlgemuth S, Stumpner A, Benda J, Ronacher B, Herz AV (2009) Timescale-invariant representation of acoustic communication signals by a bursting neuron. J Neurosci 29:2575-2580. CrossRef Medline

Creutzig F, Benda J, Wohlgemuth S, Stumpner A, Ronacher B, Herz AV (2010) Timescale-invariant pattern recognition by feedforward inhibition and parallel signal processing. Neural Comput 22:1493-1510. CrossRef Medline

de Cheveigné, A. (2005) Pitch perception models. In: Pitch (Plack C, Oxenham A, eds), pp 169-233. New York: Springer.

Drugowitsch J, Pouget A (2012) Probabilistic vs. non-probabilistic approaches to the neurobiology of perceptual decision-making. Curr Opin Neurobiol 22:963-969. CrossRef Medline

Fairhall AL, Burlingame CA, Narasimhan R, Harris RA, Puchalla JL, Berry MJ 2nd (2006) Selectivity for Multiple Stimulus Features in Retinal Ganglion Cells. J Neurophysiol 96:2724-2738. CrossRef Medline

Geffen MN, Broome BM, Laurent G, Meister M (2009) Neural encoding of rapidly fluctuating odors. Neuron 61:570-586. CrossRef Medline

Geisler WS, Diehl RL (2003) A Bayesian approach to the evolution of perceptual and cognitive systems. Cogn Sci 27:379-402. CrossRef

Geisler WS, Najemnik J, Ing AD (2009) Optimal stimulus encoders for natural tasks. J Vis 9(13):1-16. CrossRef Medline

Gold JL, Shadlen MN (2007) The neural basis of decision making. Annu Rev Neurosci 30:535-574. CrossRef Medline

Haefner RM, Gerwinn S, Macke JH, Bethge M (2013) Inferring decoding strategies from choice probabilities in the presence of correlated variability. Nat Neurosci 16:235-242. CrossRef Medline

Hennig RM, Franz A, Stumpner A (2004) Processing of auditory information in insects. Microsc Res Tech 63:351-374. CrossRef Medline

Hennig RM, Weber T (1997) Filtering of temporal parameters of the calling song by cricket females of two closely related species: a behavioral analysis. J Comp Physiol A Sens Neural Behav Physiol 180:621-630. CrossRef

Hoy RR, Hoikkala A, Kaneshiro K (1988) Hawaiian courtship songs: evolutionary innovation in communication signals of Drosophila. Science 240: 217-219. CrossRef Medline

Joris PX, Schreiner CE, Rees A (2004) Neural processing of amplitudemodulated sounds. Physiol Rev 84:541-577. CrossRef Medline

Klappert K, Reinhold K (2003) Acoustic preference functions and sexual 
selection on the male calling song in the grasshopper Chorthippus biguttulus. Anim Behav 65:225-233. CrossRef

Krahe R, Budinger E, Ronacher B (2002) Coding of a sexually dimorphic song feature by auditory interneurons of grasshoppers: the role of leading inhibition. J Comp Physiol A Neuroethol Sens Neural Behav Physiol 187: 977-985. CrossRef Medline

Kriegbaum H (1989) Female choice in the grasshopper Chorthippus biguttulus. Naturwissenschaften 76:81-82. CrossRef

Machens CK, Stemmler MB, Prinz P, Krahe R, Ronacher B, Herz AV (2001) Representation of acoustic communication signals by insect auditory receptor neurons. J Neurosci 21:3215-3227. Medline

Machens CK, Romo R, Brody CD (2005) Flexible control of mutual inhibition: a neural model of two-interval discrimination. Science 307:1121-1124. CrossRef Medline

Mitchell M (1998) An introduction to genetic algorithms (complex adaptive systems), Ed 3. Cambridge, MA: MIT.

Neri P, Levi DM (2006) Receptive versus perceptive fields from the reversecorrelation viewpoint. Vision Res 46:2465-2474. CrossRef Medline

Niven JE, Anderson JC, Laughlin SB (2007) Fly photoreceptors demonstrate energy-information trade-offs in neural coding. PLoS Biol 5:e116. CrossRef Medline

Pillow JW, Shlens J, Paninski L, Sher A, Litke AM, Chichilnisky EJ, Simoncelli EP (2008) Spatio-temporal correlations and visual signalling in a complete neuronal population. Nature 454:995-999. CrossRef Medline

Poirazi P, Brannon T, Mel BW (2003) Pyramidal neuron as two-layer neural network. Neuron 37:989-999. CrossRef Medline

Pollack GS, Hoy RR (1979) Temporal pattern as a cue for species-specific calling song recognition in crickets. Science 204:429-432. CrossRef Medline

Römer H, Seikowski U (1985) Responses to model songs of auditory neurons in the thoracic ganglia and brain of the locust. J Comp Physiol A Neuroethol Sens Neural Behav Physiol 156:845-860. CrossRef

Ronacher B, Krahe R (1998) Song recognition in the grasshopper Chorthippus biguttulus is not impaired by shortening song signals: implications for neuronal encoding. J Comp Physiol A Neuroethol Sens Neural Behav Physiol 183:729-735. CrossRef

Ronacher B, Stange N (2013) Processing of acoustic signals in grasshoppers-a neuroethological approach towards female choice. J Physiol Paris 107:41-50. CrossRef

Ronacher B, Stumpner A (1988) Filtering of behaviourally relevant temporal parameters of a grasshopper's song by an auditory interneuron. J Comp Physiol A Neuroethol Sens Neural Behav Physiol 163:517-523. CrossRef

Ryan MJ, Getz W (2000) Signal decoding and receiver evolution. An analysis using an artificial neural network. Brain Behav Evol 56:45-62. CrossRef Medline

Ryan MJ, Rand AS (2003) Sexual selection in female perceptual space: how female túngara frogs perceive and respond to complex population variation in acoustic mating signals. Evolution 57:2608-2618. CrossRef Medline

Safi K, Heinzle J, Reinhold K (2006) Species recognition influences female mate preferences in the common European grasshopper (Chorthippus biguttulus Linnaeus, 1758). Ethology 112:1225-1230. CrossRef

Schmidt A, Ronacher B, Hennig RM (2008) The role of frequency, phase and time for processing of amplitude modulated signals by grasshoppers. J Comp Physiol A Neuroethol Sens Neural Behav Physiol 194:221-233. CrossRef Medline

Schönfelder VH, Wichmann FA (2012) Sparse regularized regression identifies behaviorally-relevant stimulus features from psychophysical data. J Acoust Soc Am 131:3953-3969. CrossRef Medline

Smith EC, Lewicki MS (2006) Efficient auditory coding. Nature 439:978-982. CrossRef Medline

Stumpner A, von Helversen O (1992) Recognition of a two-element song in the grasshopper Chorthippus dorsatus (Orthoptera: Gomphocerinae). J Comp Physiol A 171:405-412.

Stumpner A, Ronacher B (1994) Neurophysiological aspects of song pattern recognition and sound localization in grasshoppers. Am Zool 34:696-705.

Uchida N, Kepecs A, Mainen ZF (2006) Seeing at a glance, smelling in a whiff: rapid forms of perceptual decision making. Nat Rev Neurosci 7:485-491. CrossRef Medline

Victor JD (2005) Analyzing receptive fields, classification images and functional images: challenges with opportunities for synergy. Nat Neurosci 8:1651-1656. CrossRef Medline

Vogel A, Hennig RM, Ronacher B (2005) Increase of neuronal response variability at higher processing levels as revealed by simultaneous recordings. J Neurophysiol 93:3548-3559. CrossRef Medline

von Helversen D (1972) Gesang des Männchens und Lautschema des Weibchens bei der Feldheuschrecke Chorthippus biguttulus (Orthoptera, Acrididae). J Comp Physiol A Neuroethol Sens Neural Behav Physiol 81:381-422. CrossRef

von Helversen O, von Helversen D (1994) Forces driving coevolution of song and song recognition in grasshoppers. Fort Zool 39:253-284.

von Helversen D, von Helversen O (1997) Recognition of sex in the acoustic communication of the grasshopper Chorthippus biguttulus (Orthoptera, Acrididae). J Comp Physiol A Neuroethol Sens Neural Behav Physiol 180:373-386. CrossRef

von Helversen D, von Helversen O (1998) Acoustic pattern recognition in a grasshopper: processing in the time or frequency domain? Biol Cybern 79:467-476. CrossRef

von Helversen D, Balakrishnan R, von Helversen O (2004) Acoustic communication in a duetting grasshopper: receiver response variability, male strategies and signal design. Anim Behav 68:131-144. CrossRef

Wang XJ (2008) Decision making in recurrent neuronal circuits. Neuron 60:215-234. CrossRef Medline

Weber F, Machens CK, Borst A (2012) Disentangling the functional consequences of the connectivity between optic-flow processing neurons. Nat Neurosci 15:441-448, S1-2. CrossRef Medline

Wilczynski W, Rand SA, Ryan MJ (1999) Female preferences for temporal order of call components in the túngara frog: a Bayesian analysis. Anim Behav 58:841-851. CrossRef Medline 